\title{
P017
}

\section{A COMPARISON OF COLOUR APPEARANCE IN VIRTUAL REALITY BETWEEN DIFFERENT SCREEN RESOLUTIONS \\ Chi-Han Ma et al.}

DOI 10.25039/x47.2020.PO17

\section{Paper accepted for the $5^{\text {th }}$ CIE Symposium on Colour and Visual Appearance}

The paper was selected by the International Scientific Committee (ISC) for presentation at the 5th CIE Symposium on Colour and Visual Appearance, Hong Kong, CN, April 21-22, 2020, which, due to the corona pandemic, could not take place. The paper has not been peer-reviewed by CIE.

\section{(C) CIE 2020}

All rights reserved. Unless otherwise specified, no part of this publication may be reproduced or utilized in any form or by any means, electronic or mechanical, including photocopying and microfilm, without permission in writing from CIE Central Bureau at the address below. Any mention of organizations or products does not imply endorsement by the CIE.

This paper is made available open access for individual use. However, in all other cases all rights are reserved unless explicit permission is sought from and given by the CIE.

CIE Central Bureau

Babenbergerstrasse 9

A-1010 Vienna

Austria

Tel.: +4317143187

e-mail: ciecb@cie.co.at

www.cie.co.at 


\title{
P017
}

\section{A COMPARISON OF COLOUR APPEARANCE BETWEEN DIFFERENT RESOLUTION IN VIRTUAL REALITY}

\author{
Ma, C. ${ }^{1}$, Huang, $\mathrm{H}^{2}{ }^{2}$, Ou, L. ${ }^{1}$ \\ ${ }^{1}$ National Taiwan University of Science and Technology, Taipei, CHINESE TAIPEI \\ ${ }^{2}$ Chihlee University of Technology, New Taipei City, CHINESE TAIPEI \\ karena6041@gmail.com
}

\begin{abstract}
This study compares colour appearance in virtual reality between different levels of display resolution. A total of 24 test colours selected from CIELAB space including 5 hue regions: red (with a hue angle of $20^{\circ}$ ), yellow $\left(90^{\circ}\right)$, green $\left(164^{\circ}\right)$, blue $\left(245^{\circ}\right)$ and purple $\left(320^{\circ}\right) .16$ observers participated in this experiment. High correlation was found between perceived lightness and CIECAM02 J, between perceived hue quadrature and CIECAM02 $\mathrm{H}$, and between perceived colourfulness and CIECAM02 $\mathrm{M}$ with a correlation coefficient of $0.88,0.99$ and 0.81 , respectively.
\end{abstract}

Keywords: Colour appearance, Virtual reality, CIECAM02

\section{Introduction}

Many types of virtual reality (VR) devices are used in our daily life, among which the most convenient one is the mobile VR headset - putting a mobile phone in the headset and then starting the VR experience anywhere. It is unclear whether there is any difference in colour appearance between colours seen in a VR environment and those in the real world, and thus it is desired to investigate the appearance of colour in VR space. In a previous study, we have conducted such a comparison between perceived colour appearance in VR and the predicted values by CIECAM02. The experimental results show high predictive performance of CIECAM02 for colour appearance in the VR environment for lightness and hue quadrature, but relatively lower correlation was found for colourfulness. We had a hypothesis that maybe the reason for this result was due to the fact that the display resolution of the mobile phone used in the experiment was too low, and that if we can increase the display resolution, we may get a different result. In that previous study, we used an iPhone 6 in the experiment, with a PPI of 326 , and this time we used an iPhone XS, with a PPI of 458, in an attempt to investigate whether the display resolution will affect the experimental results or not.

\section{Experimental Methods}

A psychophysical experiment of colour appearance was conducted in this study. We used 3DS MAX software to create a 3-dimensional interior space which was then transformed into VR images. An iPhone XS was used to present the VR images in this study. The phone had a 5.8 inch screen, with a resolution of 2436 pixels by 1125 pixels, i.e. 458 ppi. A MINISO Simple 3D VR Glasses was also used in this experiment.

\subsection{VR Environment}

The size of VR space was 220 (width) by 255 (depth) by 200 (height). All of the objects, including the walls and a desk, in this VR space had a colour of medium grey, except the three colour patches on the main wall. Three colour patches were presented side by side on the wall, with a perceived size of patches on the left side and the right side of $21.25 \mathrm{~cm}$ by $21.25 \mathrm{~cm}$. The colour patch in the middle, i.e. the test colour, had a perceived size of $37.5 \mathrm{~cm}$ by $37.5 \mathrm{~cm}$. The layout for the three colour patches is shown in Figure 1. The left patch was the reference white, with an adopted lightness value of 100 . The one on the right was the reference colourfulness, with an adopted colourfulness value of 40 . The luminance of the reference white was 
$286.53 \mathrm{~cd} / \mathrm{m}^{2}$, with $(\mathrm{x}, \mathrm{y})=(0.3177,0.3384)$. The perceived viewing distance between the observer's eyes and the main wall was $214 \mathrm{~cm}$.

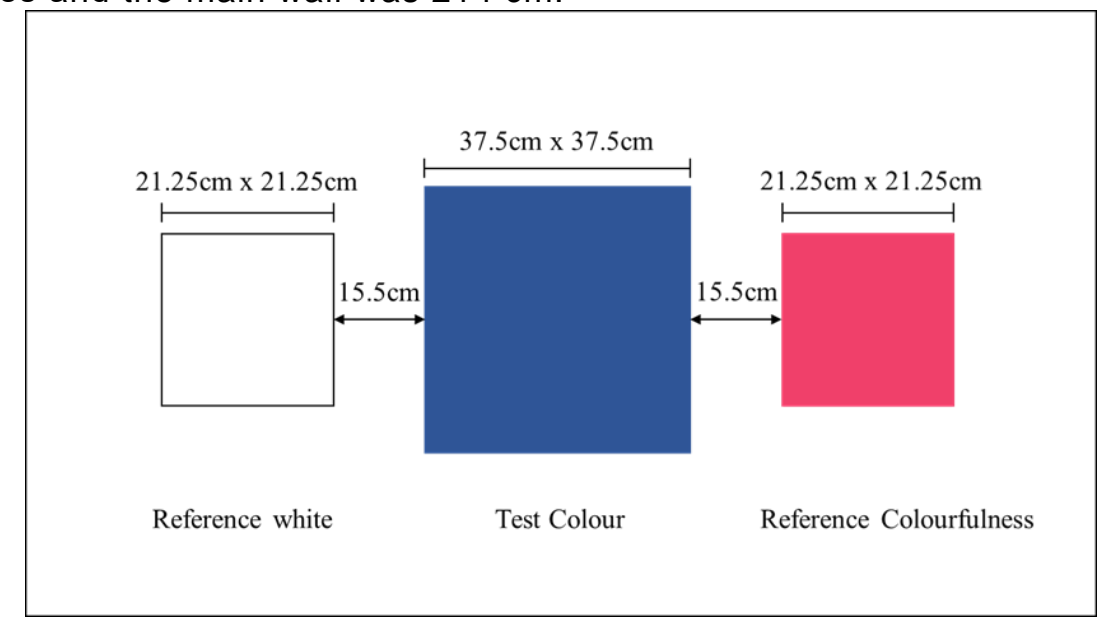

Figure 1 - The layout for colour appearance in virtual reality experiment

\subsection{Colour Samples}

24 colours were selected from CIELAB space to be used as the test colour samples in this study. Figure 2 (a)-(c) show the distribution of these test colours in CIELAB $a^{*}-b^{*}, L^{*}-a^{*}$ and $L^{*}-b^{*}$, respectively. These colours included 5 hue regions: red (with a hue angle of $20^{\circ}$ ), yellow $\left(90^{\circ}\right.$ ), green $\left(164^{\circ}\right)$, blue $\left(245^{\circ}\right)$ and purple $\left(320^{\circ}\right)$. Each hue region has 4 levels of lightness with chroma, and 4 achromatic colours. 10 of these test colours were repeated in the experiment for repeatability test. Thus, each observer made 34 visual assessments of colour appearance in the experiment. 

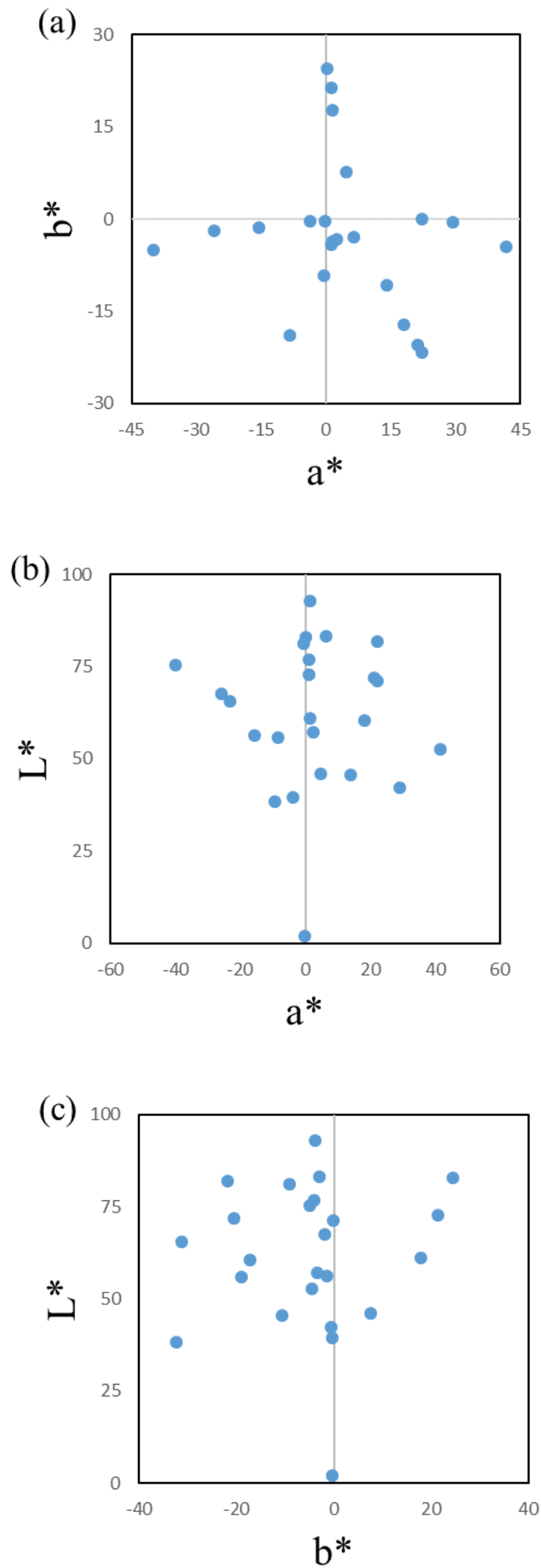

Figure 2 - The 24 test colours of this experiment plotted in (a) CIELAB $a^{*}-b^{*}$, (b) CIELAB $L^{*}-a^{*}$ and (c) CIELAB $L^{*}-b^{*}$ planes 


\subsection{Experimental Procedure}

Each observer was asked to visually assess each colour sample in terms of lightness, colourfulness and hue quadrature during the experiment. The 34 test colours, including 10 replicated colours, were presented one at a time in random order. After a test colour was assessed, a full screen of medium grey was presented for 30 seconds, after which a next test colour would be shown. The observer needed to wear the VR headset to perform the visual assessments. To prevent them from getting too tired in the eyes due to the VR experience, there was a 10-minute break after the observer has completed 17 test colours, and during the break the observer was encouraged to take off the VR headset to take a rest. The entire experiment lasted about 30 minutes for each observer.

16 observers participated in this experiment, who were all university students with normal colour vision. After the study, none of the observers reported visual discomfort.

\section{Results}

As a result, high correlation was found between perceived lightness and CIECAM02 J, with a correlation coefficient of 0.88 . High correlation was also found between perceived hue quadrature and CIECAM02 $\mathrm{H}$, with a correlation coefficient of 0.99 . However, correlation between perceived colourfulness and CIECAM02 $\mathrm{M}$ was found to be relatively lower than the other two scales, with a correlation coefficient of 0.81 . 

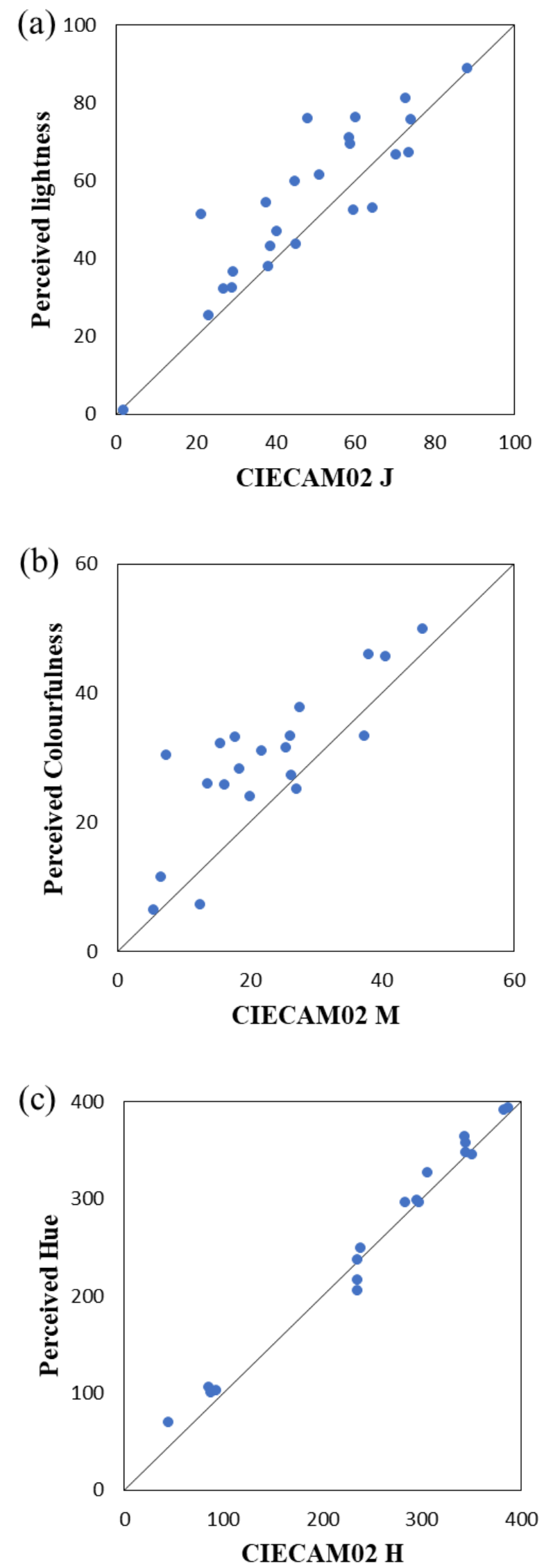

Figure 3-(a) The perceived lightness vs. CIECAM02 J, (b) perceived colourfulness vs. CIECAM02 M, (c) perceived hue quadrature vs. CIECAM02 H 


\section{Conclusion}

Comparing the experimental results with those conducted previously, both have high correlation with CIECAM02, showing good predictive performance of CIECAM02 for VR environment. Apart from hue quadrature, the perceived lightness and perceived colourfulness seem to be higher than the corresponding predicted values by CIECAM02. The scatter graph of perceived colourfulness still shows a somewhat logarithmic curve. Due to these results, maybe the resolution is not the most important factor. Future studies may look into the lighting, colours and some other conditions to increase the quality of VR experience.

\section{Acknowledgments}

This work was supported in part by the Ministry of Science and Technology, Taiwan (MOST 109-2410-H-263-001).

\section{References}

Fairchild, M.D., 2013. Color appearance models. John Wiley \&amp; Sons.

Moroney, N., Fairchild, M.D., Hunt, R.W., Li, C., Luo, M.R. and Newman, T., 2002, January. The CIECAM02 color appearance model. In Color and Imaging Conference (Vol. 2002, No. 1, pp. 23-27). Society for Imaging Science and Technology. 